\title{
The ratio of weight loss to planning target volume significantly impacts setup errors in nasopharyngeal cancer patients undergoing helical tomotherapy with daily megavoltage computed tomography
}

\author{
Wei-Hsien Hou'1, Chun-Wei Wang², Chiao-Ling Tsai², Feng-Ming Hsu², \\ Jason Chia-Hsien Cheng ${ }^{2,3,4}$

\begin{abstract}
1 Department of Radiation Oncology, City of Hope National Medical Center, Duarte, California, U.S.A
2 Division of Radiation Oncology, Departments of Oncology, National Taiwan University College of Medicine and Hospital,

Taipei, Taiwan

${ }^{3}$ Graduate Institutes of Oncology, National Taiwan University College of Medicine, Taipei, Taiwan

${ }^{4}$ Graduate Institute of Clinical Medicine, National Taiwan University College of Medicine, Taipei, Taiwan
\end{abstract}

Radiol Oncol 2016; 50(4): 427-432.

Correspondence to: Jason Chia-Hsien Cheng, M.D., Ph.D., Division of Radiation Oncology, Department of Oncology, National Taiwan University Hospital No. 7, Chung-Shan South Road, Taipei 10002, Taiwan. Phone: 886-2-2356-2842; Fax: 886-2-2331-2172; E-mail: jasoncheng@ntu.edu.tw

Disclosure: No potential conflicts of interest were disclosed.

Background. Changes in head and neck anatomy during radiation therapy (RT) produce setup uncertainties of nasopharyngeal cancer (NPC) irradiation. We retrospectively analyzed image guidance data to identify clinical predictors of setup errors.

Patients and methods. The data of 217 NPC patients undergoing definitive RT on a helical tomotherapy (HT) unit were analyzed. Factors including tumor stage, body mass index, weight loss, and planning target volume (PTV) were assessed as predictors of daily megavoltage computed tomography (MVCT) setup displacements, which were automatically registered using software.

Results. Mean daily setup displacements (in $\mathrm{mm}$ ) were $1.2 \pm 0.6,1.8 \pm 0.8,3.4 \pm 1.4$ in the medial-lateral (ML), superiorinferior (SI), and anterior-posterior (AP) directions, respectively. Mean weight loss was $4.6 \pm 3.3 \mathrm{~kg}(6.8 \pm 4.9 \%)$. Patients with weight loss $>5 \%$ had significantly larger setup displacements in the AP $(3.6 \pm 1.5 \mathrm{vs} .2 .9 \pm 1.1 \mathrm{~mm}, \mathrm{p}<0.001)$ and SI ( $1.6 \pm 0.7$ vs. $1.9 \pm 0.9 \mathrm{~mm}, p=0.01)$ direction, but not in the ML direction $(p=0.279)$. The AP setup error increased $0.06 \mathrm{~mm}(\mathrm{y}=0.055 \mathrm{x}+2.927, \mathrm{x}$ : percentage of weight loss/PTV, $y$ : AP displacement) per one percent increase in weight loss normalized to PTV.

Conclusions. Patients with weight loss $>5 \%$ and smaller PTVs, possibly because of small body frame or neck girth, were more likely to have increased setup errors in the AP direction.

Key words: nasopharyngeal cancer; intensity-modulated radiotherapy; setup errors

\section{Introduction}

Nasopharyngeal carcinoma (NPC) is a malignant neoplasm of mucosal origin. ${ }^{1}$ It has unique racial and geographic distribution with higher incidence in Southeast Asia, North Africa, and the Middle
East. ${ }^{2}$ Since the nasopharynx is located posterior to the nasal cavity and surrounded by critical structures, radical surgical resection of NPC is very challenging. ${ }^{1}$ Radiation therapy (RT) is the mainstay of treatment for NPC due to its radiosensitivity. ${ }^{3,4}$ Compared to three-dimensional conformal 
TABLE 1. Patient characteristics ( $n=217)$

\begin{tabular}{|c|c|c|}
\hline Variable & \multicolumn{2}{|c|}{ Number (\%) } \\
\hline \multicolumn{3}{|l|}{ Age } \\
\hline Median & \multicolumn{2}{|c|}{46} \\
\hline Range & \multicolumn{2}{|c|}{$17-76$} \\
\hline \multicolumn{3}{|l|}{ Sex } \\
\hline Male & 160 & (73.7) \\
\hline Female & 57 & (26.3) \\
\hline \multicolumn{3}{|l|}{ T stage } \\
\hline 1 & 97 & (44.7) \\
\hline 2 & 32 & (14.7) \\
\hline 3 & 50 & (23.0) \\
\hline 4 & 38 & (17.5) \\
\hline \multicolumn{3}{|l|}{ N stage } \\
\hline 0 & 51 & (23.5) \\
\hline 1 & 67 & (30.9) \\
\hline 2 & 67 & (30.9) \\
\hline $3 a$ & 18 & (8.3) \\
\hline $3 b$ & 15 & (6.9) \\
\hline \multicolumn{3}{|l|}{ AJCC stage } \\
\hline I & 29 & (13.4) \\
\hline ॥ & 47 & (21.7) \\
\hline III & 76 & (35.0) \\
\hline IVA & 32 & (14.7) \\
\hline IVB & 33 & (15.2) \\
\hline \multicolumn{3}{|l|}{ Chemotherapy } \\
\hline No & 27 & (12.4) \\
\hline Yes & 190 & (87.6) \\
\hline \multicolumn{3}{|l|}{ Fraction } \\
\hline 33 (2.12 Gy/fraction) & 182 & (83.9) \\
\hline 35 (2 Gy/fraction) & 35 & (16.1) \\
\hline
\end{tabular}

AJCC $=$ American Joint Committee on Cancer

radiation therapy, intensity-modulated radiation therapy (IMRT) yields superior parotid gland sparing and reduces xerostomia. ${ }^{5-7}$

IMRT delivers a conformal and steep dose gradient. Precise target localization is critical for IMRT delivery. During the course of RT to NPC, the primary tumor and surrounding anatomy commonly undergo significant volume changes secondary to tumor shrinkage and weight loss. ${ }^{8,9}$ Changes in head and neck anatomy during treatment can potentially compromise the accuracy of radiation delivery. ${ }^{8}$ The aim of image-guided radiation (i.e., image-guided radiotherapy; IGRT) is to detect and correct the set-up errors prior to treatment of head and neck cancers, including NPC. ${ }^{10-13}$

No set-up error study with large patient numbers has identified factors affecting set-up errors in radiation delivery to patients with NPC. Among IGRT modalities, helical tomotherapy with daily megavoltage computed tomography (MVCT) is the one most frequently used to evaluate and correct daily online interfraction setup errors. ${ }^{11,14}$ Using daily MVCT setup data, our aim was to determine which clinical and treatment factors (including tumor stage, body mass index [BMI], weight loss, and planning target volume [PTV]) predict setup errors in NPC patients treated with IMRT by tomotherapy.

\section{Patients and methods}

\section{Patients}

We retrospectively reviewed the clinical and RT data of 217 NPC patients (160 males and 57 females; median age 46 years [range 17-76]) who underwent definitive RT by helical tomotherapy between September 2008 and May 2013 at the National Taiwan University Hospital. Patients who received RT as salvage or with palliative intent were excluded. A total of 29 (13.4\%), 47 (21.7\%), 76 (35\%), and $65(29.9 \%)$ patients had American Joint of Cancer Committee (AJCC $7^{\text {th }}$ edition) stage I, II, III, and IV, respectively (Table 1). Concurrent cisplatin and/or tegafur-uracil (UFUR) was used in $190(87.6 \%)$ patients at the discretion of medical oncologists.

This study followed the Helsinki Declaration and complied with the ethical standard guidelines. No patient identifying information was used or reported. Our institutional review board approved this retrospective study with the approval number 201605075RINC on 6/16/16.

\section{Treatment planning}

All patients underwent CT simulation with thermoplastic mask immobilization. CT scan images of 3-mm slices were obtained with and without intravenous contrast medium. IMRT doses were delivered to three target volumes defined for each patient in 33 to 35 treatment fractions. The primary tumor and involved lymph nodes with safety margin were treated to $70 \mathrm{~Gy}$ fractionated into 2-2.12 Gy daily doses. The clinical target volume (CTV) of areas at risk for microscopic involvement (nasopharynx, retropharyngeal nodal regions, skull 
base, clivus, pterygoid fossae, parapharyngeal space, sphenoid sinus, nasal cavity, maxillary sinuses, and levels I through V cervical nodes) were treated with 59.4-64 Gy. Clinically negative parts of the lower neck were treated with 54 Gy. A margin of $3 \mathrm{~mm}$ was used for the PTV. Margins were reduced if the CTV was near critical structures including the brain stem and eyes. The prescribed dose covered $95 \%$ of the PTV. Doses to the organs at risk including brainstem, spinal cord, eyes, optic nerves, pituitary gland, parotid glands, submandibular glands, thyroid glands, cochlea, brachial plexus, and oral cavity were minimized without compromising PTV coverage.

\section{Daily MVCT and setup error}

Prior to each treatment, MVCT images were acquired after positioning the patient using wall lasers and external markings, and were reconstructed with "Normal" $(4 \mathrm{~mm}$ ) slice thickness. Setup displacements were determined with autoregistration software using the "Bone and Soft tissue" option. Setup errors of the target in the medial-lateral (ML), superior-inferior (SI), anterior-posterior (AP) directions as well as rotational errors were obtained. If a rotational displacement was larger than 3 degrees, the therapists would re-position the patient and repeat MVCT. Translational errors were corrected by automatic couch positioning.

\section{Data analyses}

Clinical and treatment factors including $\mathrm{T}$ and $\mathrm{N}$ stages, BMI, weight loss percentage, and PTV were used for data analyses. We performed the Student $t$-test and linear regression to correlate these factors with setup errors. Statistical analyses were done using the software SPSS Version 22. P values less than 0.05 were considered statistically significant.

\section{Results}

The patient cohort had an average weight ( \pm standard deviation) before RT of $67.9 \pm 13.1 \mathrm{~kg}$ (range: $40.0-128.8)$, average BMI before RT of $24.4 \pm 3.5 \mathrm{~kg} / \mathrm{m}^{2}$ (range: 16.4-40.3), mean weight loss following 6 to 7 weeks of RT of $4.6 \pm 3.3 \mathrm{~kg}$ (range: -3.8 to +14.5 ), which represented $6.8 \pm 4.9 \%$ (range: -8.3 to +18.0 ) of weight before RT, and mean PTV of $848.0 \pm 210.2$ cc (range: 415.7-1584.3). A total of 7231 MVCT images were evaluated for setup errors, with a median
TABLE 2. Setup error by clinical factors

\begin{tabular}{|c|c|c|c|}
\hline & $M L$ error & SI error & AP error \\
\hline Overall & $1.2 \pm 0.6$ & $1.8 \pm 0.8$ & $3.4 \pm 1.4$ \\
\hline \multicolumn{4}{|l|}{ T stage } \\
\hline $1,2(n=129)$ & $1.2 \pm 0.6$ & $1.8 \pm 0.9$ & $3.3 \pm 1.5$ \\
\hline $3,4(n=88)$ & $1.1 \pm 0.5$ & $1.7 \pm 0.8$ & $3.5 \pm 1.3$ \\
\hline p value & 0.241 & 0.814 & 0.414 \\
\hline \multicolumn{4}{|l|}{$\mathrm{N}$ stage } \\
\hline $0,1(n=117)$ & $1.1 \pm 0.6$ & $1.7 \pm 0.8$ & $3.4 \pm 1.3$ \\
\hline $2,3 a, 3 b(n=100)$ & $1.2 \pm 0.6$ & $1.9 \pm 0.9$ & $3.4 \pm 1.5$ \\
\hline p value & 0.133 & 0.239 & 0.890 \\
\hline \multicolumn{4}{|l|}{ BMI } \\
\hline$<25(n=138)$ & $1.2 \pm 0.6$ & $1.7 \pm 0.8$ & $3.2 \pm 1.3$ \\
\hline$\geq 25(n=79)$ & $1.2 \pm 0.5$ & $1.9 \pm 0.9$ & $3.6 \pm 1.5$ \\
\hline $\mathrm{p}$ value & 0.845 & 0.177 & 0.053 \\
\hline \multicolumn{4}{|l|}{ PTV } \\
\hline$<850 \mathrm{cc}(\mathrm{n}=113)$ & $1.2 \pm 0.7$ & $1.9 \pm 0.9$ & $3.6 \pm 1.5$ \\
\hline$\geq 850 \mathrm{cc}(\mathrm{n}=104)$ & $1.2 \pm 0.5$ & $1.6 \pm 0.7$ & $3.2 \pm 1.3$ \\
\hline p value & 0.292 & 0.021 & 0.03 \\
\hline \multicolumn{4}{|l|}{ Weight loss } \\
\hline$\leq 5 \%(n=84)$ & $1.1 \pm 0.4$ & $1.6 \pm 0.7$ & $2.9 \pm 1.1$ \\
\hline$>5 \%(n=133)$ & $1.2 \pm 0.6$ & $1.9 \pm 0.9$ & $3.6 \pm 1.5$ \\
\hline $\mathrm{p}$ value & 0.279 & 0.010 & $<0.001$ \\
\hline
\end{tabular}

$\mathrm{AP}=$ anterior-posterior; $\mathrm{BMI}=$ body mass index; $\mathrm{ML}=$ medial-lateral; PTV = planning target volume; $\mathrm{SI}=$ superior-inferior.

TABLE 3. Setup errors by the specified threshold

\begin{tabular}{lccc}
\hline & ML error (\%) & SI error (\%) & AP error (\%) \\
\hline$<1.0 \mathrm{~mm}$ & $3549(49.1)$ & $2471(34.2)$ & $525(7.3)$ \\
$\mathbf{1 . 0} 1.9 \mathrm{~mm}$ & $2315(32.0)$ & $2076(28.7)$ & $939(13.0)$ \\
$\mathbf{2 . 0} 2.9 \mathrm{~mm}$ & $925(12.8)$ & $1390(19.2)$ & $1492(20.6)$ \\
$\mathbf{3 . 0} \mathbf{3 . 9} \mathrm{mm}$ & $314(4.3)$ & $729(10.1)$ & $1800(24.9)$ \\
$\mathbf{4 . 0} \mathbf{4 . 9} \mathrm{mm}$ & $85(1.2)$ & $330(4.6)$ & $1310(18.1)$ \\
$\geq \mathbf{5 . 0} \mathrm{mm}$ & $45(0.6)$ & $237(3.3)$ & $1167(16.1)$ \\
\hline
\end{tabular}

$\mathrm{AP}=$ anterior-posterior; $\mathrm{ML}=$ medial-lateral; $\mathrm{SI}$ = superior-inferior

number of 33 (range: 33-35) MVCT images per patient.

Mean daily setup displacements (in $\mathrm{mm}$ ) were $1.2 \pm 0.6,1.8 \pm 0.8$, and $3.4 \pm 1.4$ in the ML, SI, and AP directions, respectively (Table 2). The displacement was significantly larger in the AP direction than in the ML $(p<0.001)$ and SI $(p<0.001)$ directions. The displacement was also significantly 


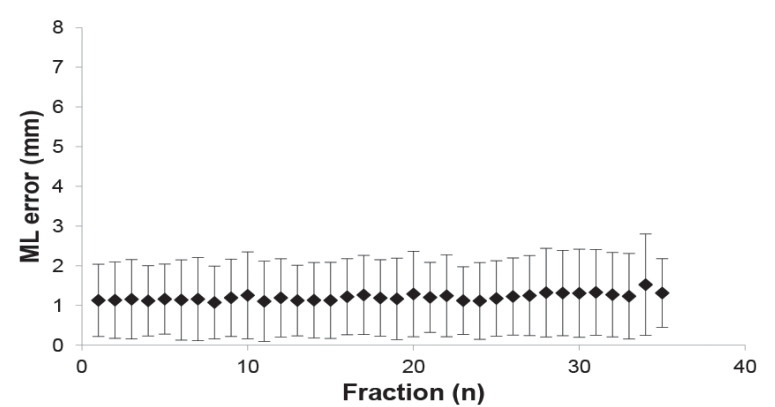

FIGURE 1. Setup error of the medial-lateral (ML) direction at each treatment fraction.

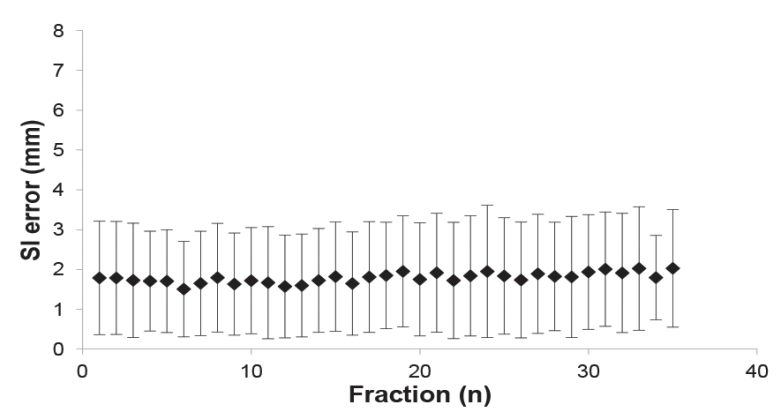

FIGURE 2. Setup error of the superior-inferior (SI) direction at each treatment fraction.

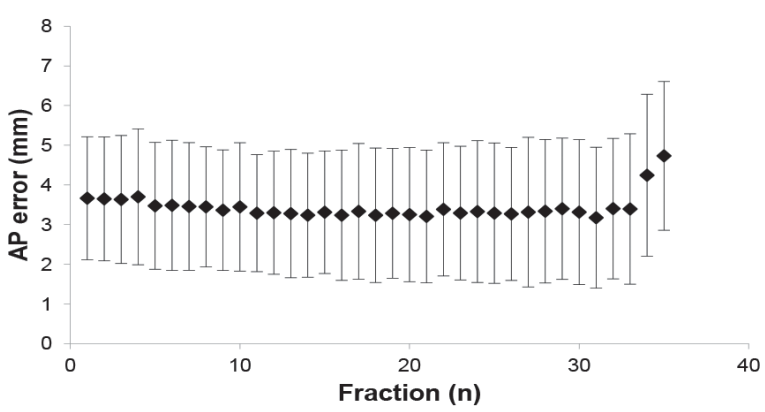

FIGURE 3. Setup error of the anterior-posterior (AP) direction at each treatment fraction.

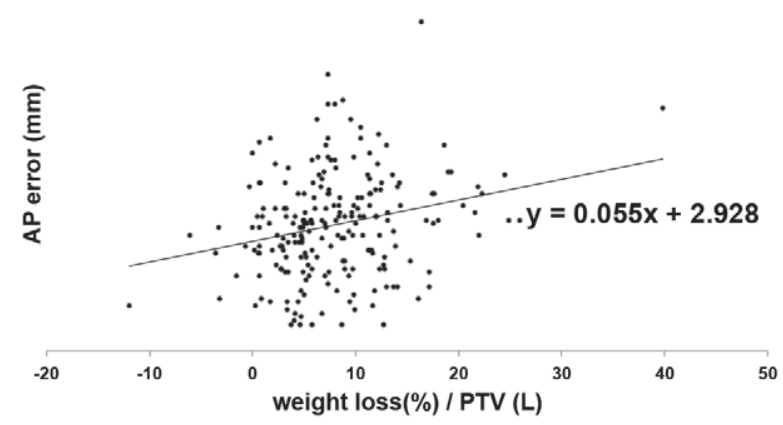

FIGURE 4. Linear regression graph of anterior-posterior (AP) setup error and weight loss normalized by planning target volume (PTV) $\left(R^{2}=0.059, p<0.001\right)$. larger in SI direction than in the ML direction $(\mathrm{p}<0.001)$. Setup errors greater than $3 \mathrm{~mm}$ occurred for $6.1 \%, 18.0 \%$, and $59.1 \%$ of treatment fractions in the ML, SI, and AP directions, respectively (Table 3). There was a trend of increased setup errors toward the end of treatment course, especially in the AP direction (Figures 1-3).

Next, we determined whether $\mathrm{T}$ stage, $\mathrm{N}$ stage, BMI, PTV, or weight loss were factors affecting setup errors using the Student $t$-test. Setup displacements in all directions were not significantly different between patients with different $\mathrm{T}$ stage (1-2 vs. $3-4)$ and $N$ stage (0-1 vs. 2-3). There was a strong trend indicating that patients with larger BMI $(\geq 25)$ before RT had an increased setup displacement in the AP direction $(3.6 \pm 1.5 \mathrm{~mm}$ vs. $3.2 \pm$ $1.3 \mathrm{~mm}, \mathrm{p}=0.053$ ) during treatment. Smaller PTV $(<850 \mathrm{cc})$ was associated with larger setup displacement in the ML direction $(1.9 \pm 0.9$ vs. $1.6 \pm 0.7 \mathrm{p}=0.021)$ and AP direction $(3.6 \pm 1.5 \mathrm{~mm}$ vs. $3.2 \pm 1.3 \mathrm{~mm}, \mathrm{p}=0.03$ ). Patients with weight loss $>5 \%$ had a significantly larger setup displacement in the $\mathrm{AP}(3.6 \pm 1.5 \mathrm{~mm}$ vs. $2.9 \pm 1.1 \mathrm{~mm}, \mathrm{p}<0.001)$, and SI $(1.9 \pm 0.9 \mathrm{~mm} v s .1 .6 \pm 0.7 \mathrm{~mm} \mathrm{p}=0.01)$ directions but not in the ML $(\mathrm{p}=0.279)$ direction.

Treatment volume strongly depended on gross disease volume, neck girth, and target margins. To control the effect of treatment volume on weight loss, we normalized the amount of weight loss to PTV. We calculated the normalized weight loss by the ratio of the percentage of weight loss over PTV (cc). As shown in Figure 4, normalized weight loss correlated significantly with setup displacement in the AP direction $\left(R^{2}=0.059, p<0.001\right)$. For every one percent increase in weight loss normalized to PTV, AP setup error was increased by $0.06 \mathrm{~mm}$ ( $\mathrm{y}=$ $0.055 x+2.927, x$ : percentage of weight loss/PTV, $y$ : AP setup displacement).

\section{Discussion}

Setup uncertainties greatly impact the accuracy of intensity-modulated radiation delivery. CT image guidance is routinely used to correct setup errors prior to radiation delivery. A prior small study of ten nasopharynx and nasal cavity cancer patients treated with RT has shown a large setup error of $3.6 \pm 1.0 \mathrm{~mm}$ without daily CT image guidance. ${ }^{11}$ In our study, the daily set-up variations were assessed in NPC patients treated with definitive IMRT delivered by helical tomotherapy. A large setup displacement in the AP direction with error greater than $3.0 \mathrm{~mm}$ occurred for $59.1 \%$ of treat- 
ment fractions. The nasopharynx is situated immediately anterior to the clivus and brainstem. For nasopharyngeal cancer with clival involvement, the posterior margin and PTV expansion was often small or absent in order to avoid the brainstem. Therefore, our study suggests that daily IGRT is crucial in ensuring adequate coverage of nasopharyngeal gross tumor volume and protection of the brainstem.

To our knowledge, our study produced the largest dataset of daily setup errors by helical tomotherapy during NPC RT. Helical tomotherapybased MVCT uses the same X-ray source for image acquisition and treatment. ${ }^{15}$ No surrogate telemetry systems are required to register image space to treatment space. In contrast, the kilovoltage $(\mathrm{kV})$ cone-beam computed tomography (CBCT) on a linear accelerator can produce systematic errors due to misalignment. Compared with MVCT, kV CBCT offers superior spatial and contrast resolution because of less Compton scattering and pair production interaction with its $\mathrm{kV}$ photon source. ${ }^{16}$ Nevertheless, helical tomotherapy MVCT can provide sufficient contrast to delineate many soft tissue structures. ${ }^{17}$ The cone beam geometry of CBCT systems can generate a larger scattered radiation component affecting image quality indices including homogeneity, contrast, and noise in the reconstructed CBCT images. ${ }^{18}$ Furthermore, MVCT scan contrast is linearly related to the electron density of the material imaged and is not associated with scatter artifacts produced by dental prosthesis. ${ }^{17}$ Given these potential shortcomings of $\mathrm{kV} \mathrm{CBCT}$, helical tomotherapy MVCT might be a more suitable choice for daily IGRT.

NPC is a lymphoid-rich neoplasm that is radiation-sensitive and undergoes dramatic shrinkage in response to radiation..$^{19}$ Shrinkage of the gross tumor volume can lead to anatomy shifts and increased setup errors. A previous study using electronic portal imaging in 20 patients undergoing definitive IMRT showed setup displacements of $>3 \mathrm{~mm}$ occurred more frequently in patients with bulky nodal disease. ${ }^{13}$ In contrast to the previous finding, our data indicated no worsening of setup errors in patients with advanced $\mathrm{T}$ stage or N stage NPC. Furthermore, weight loss greatly impacts setup error magnitude, and is consistent with previous findings in patients treated with head and neck RT. 13,20,21 Weight loss during RT for head and neck cancers is multi-factorial. Ionizing radiation, along with concurrent systemic chemotherapy, induces acute mucositis and taste change. ${ }^{6}$ Radiation-induced xerostomia contributes to swal- lowing difficulty. Inadvertent radiation dose to the brainstem and vestibule as well as platinum-based chemotherapy can induce intractable nausea. ${ }^{22}$ These factors combine to contribute to severe malnourishment and weight loss during NPC RT.

Several potential strategies can minimize weight loss-induced setup errors during RT. Rigorous weight monitoring and nutrition support with enteral feeding can ameliorate malnutrition and help preserve head and neck muscle mass and subcutaneous fat. ${ }^{23}$ Aggressive management of mucositis and nausea with supportive care can alleviate discomfort during food intake. ${ }^{24}$ Optimization of radiation treatment planning can help reduce mucositis and nausea by minimizing the dose to the mucosal surface and brainstem. ${ }^{22,25}$ As observed in our study, there is a trend toward enlarged setup errors with increasing number of fractions. The use of a shorter radiation treatment course with altered fractionation scheme may also help reduce setup errors. Adaptive RT can potentially decrease radiation dose to normal organs and thereby reduce xerostomia and nausea. ${ }^{26-30}$

Volume of irradiated normal tissue is correlated with RT toxicity. Size of the PTV can be a crude predictor of acute RT toxicity. ${ }^{31,32}$ However, we found that patients with larger PTV actually had smaller setup errors in the SI and AP directions. Our image guidance procedure allows the observers to apply additional shifts to ensure adequate target coverage following automatic correction of setup displacements. It is possible that the larger PTV achieves smaller setup error by reducing these shifts. In our study, weight loss normalized to PTV accounts for the observed effect of PTV on setup errors. Importantly, weight loss normalized to PTV is a more precise measure of "tissue density loss" and correlates better with setup errors due to weight change.

Our study had several limitations including the absence of post-treatment images that can reveal the extent of intrafraction displacement. Nonetheless, a prior study that employs post-treatment cone-beam CTs has shown a small intrafraction motion of $1.2 \mathrm{~mm}$ during RT delivery to NPC with standard thermoplastic mask immobilization..$^{33}$ Our study also lacks treatment toxicity data that would further determine the causes of weight loss. The retrospective nature of this analysis poses the risks of patient selection bias and uncontrolled confounding factors. Despite these limitations, using our large daily MVCT dataset, we were able to identify weight loss as an independent risk factor for setup errors. Future prospective study is need- 
ed to determine if weight loss intervention reduces interfraction setup errors in NPC RT.

In conclusion, weight loss is an independent risk factor for setup error during RT delivery to NPC. Patients with a moderate weight loss of more than $5 \%$ may be susceptible to increased interfraction $\mathrm{AP}$ and SI setup errors. Ameliorating weight loss during RT to NPC by close dietary monitoring and appropriate interventions may improve target coverage and reduce treatment toxicity by reducing the frequency and magnitude of setup errors.

\section{References}

1. Wei WI, Sham JS. Nasopharyngeal carcinoma. Lancet 2005; 365: 2041-54.

2. Yu MC, Yuan JM. Epidemiology of nasopharyngeal carcinoma. Semin Cance Biol 2002; 12: 421-9.

3. Lee N, Harris J, Garden AS, Straube W, Glisson B, Xia P, et al. Intensitymodulated radiation therapy with or without chemotherapy for nasopharyngeal carcinoma: radiation therapy oncology group phase II trial 0225. $J$ Clin Oncol 2009; 27: 3684-90.

4. Tham IW, Hee SW, Yeo RM, Salleh PB, Lee J, Tan TW, et al. Treatment of nasopharyngeal carcinoma using intensity-modulated radiotherapy-the national cancer centre Singapore experience. Int J Radiat Oncol Biol Phys 2009; 75: 1481-6.

5. Kam MK, Leung SF, Zee B, Chau RM, Suen JJ, Mo F, et al. Prospective randomized study of intensity-modulated radiotherapy on salivary gland function in early-stage nasopharyngeal carcinoma patients. J Clin Oncol 2007; 25: 4873-9.

6. Pow EH, Kwong DL, McMillan AS, Wong MC, Sham JS, Leung LH, et al. Xerostomia and quality of life after intensity-modulated radiotherapy vs. conventional radiotherapy for early-stage nasopharyngeal carcinoma: initial report on a randomized controlled clinical trial. Int J Radiat Oncol Biol Phys 2006; 66: 981-91.

7. Songthong A, Chakkabat C, Kannarunimit D, Lertbutsayanukul C. Efficacy of intensity-modulated radiotherapy with concurrent carboplatin in nasopharyngeal carcinoma. Radiol Oncol 2015; 49: 155-62.

8. Cheng HC, Wu VW, Ngan RK, Tang KW, Chan CC, Wong KH, et al. A prospective study on volumetric and dosimetric changes during intensity-modulated radiotherapy for nasopharyngeal carcinoma patients. Radiother Oncol 2012; 104: 317-23.

9. Chen C, Fei Z, Chen L, Bai P, Lin X, Pan J. Will weight loss cause significant dosimetric changes of target volumes and organs at risk in nasopharyngeal carcinoma treated with intensity-modulated radiation therapy? Med Dosim 2014; 39: 34-7.

10. Wang J, Bai S, Chen N, Xu F, Jiang X, Li Y, et al. The clinical feasibility and effect of online cone beam computer tomography-guided intensity-modulated radiotherapy for nasopharyngeal cancer. Radiother Oncol 2009; 90: 221-7.

11. Sheng K, Chow MC, Hunter G, Larner JM, Read PW. Is daily CT image guidance necessary for nasal cavity and nasopharyngeal radiotherapy: an investigation based on helical tomotherapy. J App/ Clin Med Phys 2008; 9: 2686.

12. Leung SW, Lee TF. Treatment of nasopharyngeal carcinoma by tomotherapy: five-year experience. Radiat Oncol 2013; 8: 107.

13. Mongioj V, Orlandi E, Palazzi M, Deponti E, Marzia F, Stucchi C, et al. Set-up errors analyses in IMRT treatments for nasopharyngeal carcinoma to evaluate time trends, PTV and PRV margins. Acta Oncol 2011; 50: 61-71.

14. Tome WA, Jaradat HA, Nelson IA, Ritter MA, Mehta MP. Helical tomotherapy: image guidance and adaptive dose guidance. Front Radiat Ther Oncol 2007; 40: 162-78

15. Yartsev S, Kron T, Van Dyk J. Tomotherapy as a tool in image-guided radiation therapy (IGRT): theoretical and technological aspects. Biomed Imaging Interv J 2007; 3: e16.
16. Yadav P, Ramasubramanian V, Paliwal BR. Feasibility study on effect and stability of adaptive radiotherapy on kilovoltage cone beam CT. Radiol Oncol 2011; 45: 220-6.

17. Meeks SL, Harmon JF, Jr., Langen KM, Willoughby TR, Wagner TH, Kupelian PA. Performance characterization of megavoltage computed tomography imaging on a helical tomotherapy unit. Medi Phys 2005; 32: 2673-81.

18. Chan M, Yang J, Song Y, Burman C, Chan P, Li S. Evaluation of imaging performance of major image guidance systems. Biomed Imaging Interv J 2011; 7: e11.

19. Jayasurya A, Bay BH, Yap WM, Tan NG. Lymphocytic infiltration in undifferentiated nasopharyngeal cancer. Arch Otolaryngol Head Neck Surg 2000; 126: $1329-32$.

20. Johansen J, Bertelsen A, Hansen CR, Westberg J, Hansen O, Brink C. Set-up errors in patients undergoing image guided radiation treatment. Relationship to body mass index and weight loss. Acta Oncol 2008; 47: 1454-8.

21. Lai YL, Yang SN, Liang JA, Wang YC, Yu CY, Su CH, et al. Impact of bodymass factors on setup displacement in patients with head and neck cancer treated with radiotherapy using daily on-line image guidance. Radiat Oncol 2014; 9: 19.

22. Lee $\mathrm{VH}, \mathrm{Ng} \mathrm{SC}$, Leung TW, Au GK, Kwong DL. Dosimetric predictors of radiation-induced acute nausea and vomiting in IMRT for nasopharyngeal cancer. Int J Radiat Oncol Biol Phys 2012; 84: 176-82.

23. Beaver ME, Matheny KE, Roberts DB, Myers JN. Predictors of weight loss during radiation therapy. Otolaryngol Head Neck Surg 2001; 125: 645-8.

24. Lalla RV, Bowen J, Barasch A, Elting L, Epstein J, Keefe DM, et al. MASCC/ ISOO clinical practice guidelines for the management of mucositis secondary to cancer therapy. Cancer 2014; 120: 1453-61.

25. Lee FK, Yip CW, Cheung FC, Leung AK, Chau RM, Ngan RK. Dosimetric difference amongst 3 techniques: TomoTherapy, sliding-window intensity-modulated radiotherapy (IMRT), and RapidArc radiotherapy in the treatment of late-stage nasopharyngeal carcinoma (NPC). Med Dosim 2014; 39: 44-9.

26. Yang H, Hu W, Wang W, Chen P, Ding W, Luo W. Replanning during intensity modulated radiation therapy improved quality of life in patients with nasopharyngeal carcinoma. Int J Radiat Oncol Biol Phys 2013; 85: e47-54.

27. Fung WW, Wu VW, Teo PM. Developing an adaptive radiation therapy strategy for nasopharyngeal carcinoma. J Radiat Res 2014; 55: 293-304.

28. Chen AM, Daly ME, Cui J, Mathai M, Benedict S, Purdy JA. Clinical outcomes among patients with head and neck cancer treated by intensity-modulated radiotherapy with and without adaptive replanning. Head Neck 2014; 36 1541-6.

29. Ren G, Xu SP, Du L, Feng LC, Qu BL, Liu HX, et al. Actual anatomical and dosimetric changes of parotid glands in nasopharyngeal carcinoma patients during intensity modulated radiation therapy. Biomed Res Int 2015; 2015: 670327.

30. Yao WR, Xu SP, Liu B, Cao XT, Ren G, Du L, et al. Replanning Criteria and Timing Definition for Parotid Protection-Based Adaptive Radiation Therapy in Nasopharyngeal Carcinoma. Biomed Res Int 2015; 2015: 476383.

31. Lin YW, Chen CC, Lin LC, Lee SP. The impact of reduced-volume, intensitymodulated radiation therapy on disease control in nasopharyngeal carcinoma. Plos One 2015; 10: e0125283.

32. Munbodh R, Jackson A, Bauer J, Schmidtlein CR, Zelefsky MJ. Dosimetric and anatomic indicators of late rectal toxicity after high-dose intensity modulated radiation therapy for prostate cancer. Med Phys 2008; 35: 2137-50.

33. $\mathrm{Lu} \mathrm{H}$, Lin $\mathrm{H}$, Feng $\mathrm{G}$, Chen J, Shu L, Pang $\mathrm{Q}$, et al. Interfractional and intrafractional errors assessed by daily cone-beam computed tomography in nasopharyngeal carcinoma treated with intensity-modulated radiation therapy: a prospective study. J Radiat Res 2012; 53: 954-60. 